15) Levich, V. G.: "Physicochemical Hydrodynamics", p. 549, Prentice-Hall, Englewood Cliffs (1962).

16) Licht, W. and W. F. Pansing: Ind. Eng. Chem., 45, 1885 (1953).

17) Losev, B. D. and A. S. Zheleznyak: Theor. Found. Chem. Eng., 10, 599 (1976).

18) Marsh, B. D. and W. J. Heideger: Ind. Eng. Chem., Fundam., 4, 129 (1965).

19) Nakashio, F., K. Inoue and K. Kondo: Kagaku Kögaku, 42, 182 (1978).

20) Okada, S., S. Yoshizawa, F. Hine and K. Asada: Denki Kagaku, 25, 562 (1957).

21) Popovich, A. T., R. E. Jervis and O. Trass: Chem. Eng. Sci., 19, 357 (1964).

22) Rajan, S. M. and W. J. Heideger: AIChE J., 17, 202 (1971).

23) Rosner, D. E.: ibid., 9, 321 (1963).
24) Sato, T., T. Yoshino and T. Nakamura: J. Inorg. Nuc. Chem., 40, 1571 (1978).

25) Shirotsuka, T. and M. Sano: Kagaku Kogaku, 32, 687 (1968).

26) Simpkins, P. G.: Int. J. Heat Mass Transfer, 8, 99 (1965).

27) Skelland, A. H. P. and S. S. Minhas: AIChE J., 17, 1316 (1971).

28) Tarasov, V. V., A. V. Fomin and G. A. Yagodin: Soviet Radiochemistry, 19, 628 (1977).

29) Vandegrift, G. F. and E. P. Horwitz: J. Inorg. Nucl. Chem., 39, 1425 (1977).

30) Vetter, K. J.: "Electrochemical Kinetics, Theoretical Aspects", p. 268, Academic Press, New York (1967).

31) Walia, D. S. and D. Vir: Chem. Eng. Sci., 31, 525 (1976), Chem. Eng. J., 12, 133 (1976).

\title{
EXTRACTION KINETICS OF COPPER WITH HYDROXYOXIME EXTRACTANT
}

\author{
ISAO KOMASAWA, TSUTAO OTAKE \\ AND TOSHIKAZU MURAOKA \\ Department of Chemical Engineering, Osaka University, \\ Toyonaka 560
}

\begin{abstract}
The kinetics of the extraction of copper from sulphate media with a hydroxyoxime chelating extractant was studied using a single-drop technique. A simple kinetic scheme involving the species adsorbed at the aqueous-organic interface was proposed. Assuming the extreme conditions of chemical reaction control to apply, the extraction rates were found to be directly proportional to both copper (II) ion and monomeric oxime concentrations and inversely proportional to hydrogen ion concentration. These results support the reaction scheme proposed.

The ratio of the rate constant for the $n$-heptane diluent system to that for the toluene diluent system was found to be as large as 20 . This effect is thought to occur owing to the interfacial population density of adsorbed species and the free monomeric oxime content in the organic phase.
\end{abstract}

\section{Introduction}

Many studies have previously reported the kinetic behaviour of heterogeneous reactions involving a metal solute in liquid-liquid systems. For the extraction of copper with a hydroxyoxime extractant, investigations have been carried out using a singledrop technique ${ }^{10,13)}$, an AKUFVE mixer $^{4}$, a conventional mixer ${ }^{11}$, and a stirred transfer cell $^{2,6)}$. Several critical reviews have also been published ${ }^{3,5}$. There are, however, differences between the forms of the observed rate expressions according to the different techniques and concentration levels chosen for the kinetic study ${ }^{5}$.

Received July 61979 . Correspondence concerning this article should be addressed to I. Komasawa. T. Muraoka is now with Nissan Chemical Industries Ltd., Toyama 939-27.
Two problems may be implicitly involved and these must be solved to reconcile these differences. One concerns the active species during the process of extraction. In the previous study ${ }^{7}$, it was demonstrated that the active species involved were the copper (II) ion in the aqueous phase and the monomeric oxime in the organic phase, rather than the total copper or total oxime. The second concerns the relative importance of chemical and diffusional resistances in the overall extraction rate. For extraction to proceed, the active species in each bulk phase must find their way, as a whole, into a region comprising the aqueous phase, a zone on the aqueous side in the interfacial zone, or at least, to the interface, where the chelating reaction is assumed to take place.

A systematic investigation of the kinetics, or at least the rate of chemical reaction, may be of great impor- 
tance in an understanding of the underlying process mechanism. In the present study, a simple kinetic scheme is proposed, based on the nature of the hydroxyoxime extractant. The extraction rates were measured using a single-drop technique. Assuming the extreme conditions of negligible contribution by diffusional resistance to apply, the extraction rates were correlated as functions of the concentrations of the active species and compared with predictions from the proposed reaction scheme. Finally, the effect of the diluent on the kinetics is discussed.

\section{Experiment}

Aqueous solutions were prepared by dissolving copper sulphate in deionized water, to which a small amount of sulfuric acid was added as a $\mathrm{pH}$ adjustant. Organic solutions were prepared on a volumetric basis by diluting commercial LIX65N as received or anti 2-hydroxy-5-nonylbenzophenone oxime (the anti HNBPO, active ingredient in LIX65N) in $n$-heptane or toluene. Analytical procedures for the aqueous and organic solutions were the same as those described in the previous publication ${ }^{7}$.

The apparatus, shown in Fig. 1, is similar to that reported for a single-drop mass transfer study ${ }^{9}$. The organic-phase drops were formed through a flat-ended stainless steel needle and flowed upward through the surrounding aqueous copper sulphate solution. Drop throughput was held constant at $71 \pm 3 \mathrm{drops} / \mathrm{min}$ in all runs. The drops finally coalesced at position $\mathbf{A}$ in Fig. 1, where the diameter of the collection part of the tube was reduced to $0.5 \mathrm{~cm}$ in order to minimize end effects due to coalescence. The outlet dispersed phase then passed through the capillary tube to a small sampling flask. After steady state was attained, the sampling of loaded organic solution was started and continued for 5 to $15 \mathrm{~min}$. The drop volume was calculated from the number of drops and the resulting weight of sampled organic solution. The time of contact between the organic drops and the aqueous solution was varied by the use of columns of three differing lengths $(60,120$ and $215 \mathrm{~cm})$. The temperature was maintained at $25 \pm 0.1^{\circ} \mathrm{C}$.

The glassware was cleaned with acetone and then with a surfactant, Dacon 90 . It was then thoroughly flushed with running water for a prolonged period and finally rinsed with deionized water. A freshly prepared needle was used in each run to maintain the drop volume constant. Preliminary checks showed that this cleaning procedure and the use of freshly prepared needles yielded satisfactory, reproducible data both for the drop volume and the measured extraction rate.

Several sets of runs were carried out in which only the drop diameter was varied by the use of needles of

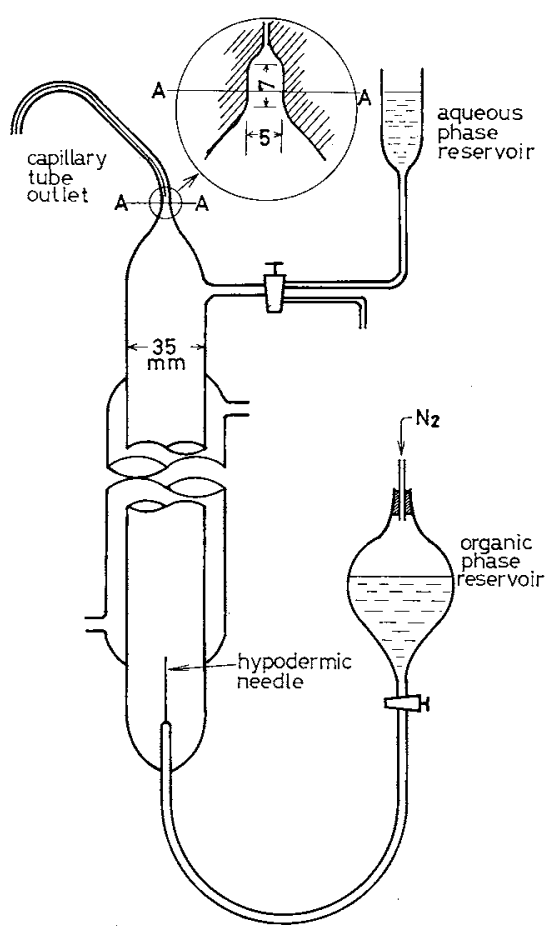

Fig. 1 Single-drop apparatus

two differing sizes, $0.3 \mathrm{~mm}$ and $1.5 \mathrm{~mm}$ outside diameter. For all other runs, the needles used were $0.5 \mathrm{~mm}$ diameter for $n$-heptane and $0.4 \mathrm{~mm}$ for toluene solutions. The mean values obtained for the drop diameters and the corresponding terminal rise velocities were $2 \times 10^{-1} \mathrm{~cm}$ and $11 \mathrm{~cm} / \mathrm{sec}$ for $n$-heptane and $2.5 \times 10^{-1} \mathrm{~cm}$ and $7.6 \mathrm{~cm} / \mathrm{sec}$ for toluene solutions.

The copper concentration in the aqueous feed solution was in the range of $1 \times 10^{-2}-2 \times 10^{-1} \mathrm{~mol} / \mathrm{dm}^{3}$ and the hydrogen ion concentration was in the range of $4 \times 10^{-5}-8.5 \times 10^{-2} \mathrm{~mol} / \mathrm{dm}^{3}(\mathrm{pH} ; 1.07-4.4)$. Copper sulphate is not completely dissociated, forming ionpair complex. The free copper (II) ion concentration was calculated from the total copper concentration and the $\mathrm{pH}$ values, using the dissociation constants ${ }^{14 \text { ( }}$ $\left[\mathrm{Cu}^{2+}\right]\left[\mathrm{SO}_{4}^{2-}\right] /\left[\mathrm{CuSO}_{4}\right]=2 \times 10^{-3}$ and $\left[\mathrm{H}^{+}\right]\left[\mathrm{SO}_{4}^{2-}\right] /$ $\left[\mathrm{HSO}_{4}^{-}\right]=7.7 \times 10^{-3} \mathrm{~mol} / \mathrm{dm}^{3}$. The oxime concentration was in the range $8.6 \times 10^{-3}-1.72 \times 10^{-1} \mathrm{~mol} / \mathrm{dm}^{3}$ in both diluent systems. The monomeric oxime concentration was calculated using the dimerization constants $K_{m}=120$ for $n$-heptane and $K_{m}=2 \mathrm{dm}^{3} / \mathrm{mol}$ for toluene diluent, i.e., it was calculated according to Eqs. (7) and (8) in the previous publication ${ }^{7}$.

\section{Results and Discussion}

\section{1 Expression of extraction rate}

When the amount of copper extracted per drop, $V \cdot \overline{C_{\mathrm{CuR}_{2}}}$, was plotted against the contact time $t$, a straight line was obtained. The slope of the resultant line gives the rate of extraction, i.e., $R=V \cdot d \overline{\mathrm{CuR}_{2}} / d t$ $[\mathrm{mol} / \mathrm{sec}]$. The line did not pass the origin, but had some intercept value. This was regarded as a measure 


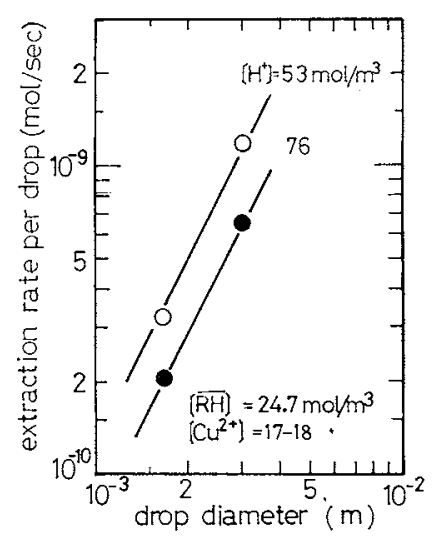

Fig. 2 Effect of drop diameter on extraction rate per drop

of the end effect in drop formation and coalescence, and is not considered further. For a limited contact time between the two phases, the organic-phase copper concentration was at most $10 \%$, and in the majority of cases less than $5 \%$, of the equilibrium value. Hence, the contribution of reverse extraction may be taken as negligible.

For the runs in which only drop diameter was varied, the rate of extraction was found to be proportional to the 2 nd power of the drop diameter, as shown in Fig. 2. The rates of extraction in this reaction system are expressed in moles of copper extracted into the drops per unit interfacial area per unit time as follows:

$$
N=R / A=(V / A)\left(d \overline{C_{\mathrm{CuR}_{2}}} / d t\right) \quad\left[\mathrm{mol} / \mathrm{m}^{2} \cdot \mathrm{sec}\right]
$$

\section{2 Diffusional resistance}

The chelating reaction may take place either in the aqueous phase (in a narrow zone adjacent to the interface or in bulk phase) or at the interface. The reactive species in the bulk phases must diffuse to the reaction zone and the product (hydrogen ion and copper complex) away from the zone. The observed copper extraction rates shown in Fig. 2 were compared with the maximum possible values of the simple physical transfer rate of the oxime. The maximum values are expressed as $N^{\prime}=\bar{k}_{\mathrm{RH}} \times[\overline{\mathrm{R}} \overline{\mathrm{H}}]$, where $\overline{k_{\mathrm{RH}}}$ and $[\overline{\mathrm{RH}}]$ are the mass transfer coefficient and the concentration of the oxime in the organic phase, respectively. The ratio of the copper flux observed to the maximum transfer rate, $N / N^{\prime}$, can be equated with the relative difference of the concentrations of the species between the interface and bulk phase. The value of $\overline{k_{\mathrm{RH}}}$ is in the range of $10^{-5}-10^{-4} \mathrm{~m} / \mathrm{sec}^{9}$. The maximum rates thus calculated were found to be one or two orders of magnitude greater than the observed rates shown in Fig. 2 and all the observed rates obtained at high acidity $(\mathrm{pH}<2.1)$ in the present runs. When applying the diffusion model accompanied by chemical reaction to the present system, the present heterogeneous reaction should be in the regime of chemical reaction control, and the rate should be expressed in terms of unit volume of the aqueous phase. The observed rates, however, were found to be proportional to the interfacial area between two phases. This suggests that the present reaction is an interfacial one. The diffusional resistance in copper extraction is taken as the resistance of transfer of the reactive species and the products to and from the interface.

The copper extraction rate is known to be determined by the concentrations of at least three species (copper (II) ion, hydrogen ion and monomeric oxime). More systematic evaluation is therefore necessary regarding the contribution by the diffusional resistance to the apparent copper extraction rates, and this will be done in a subsequent publication ${ }^{8)}$.

\section{3 Reaction scheme}

All hydroxyoxime extractants are interfacially active. It was found in the investigation of the effect of diluents on the kinetics that the variation in interfacial area per oxime molecule caused by changing the diluent had a direct effect on the rate of copper extraction ${ }^{3}$. This suggests that the probable reaction mechanism involves the adsorbed species. The rate-controlling step is considered to be the formation of a neutral complex at the interface, according to the following reaction scheme:

$$
\begin{aligned}
& \overline{\mathrm{RH}}_{\mathrm{int}} \rightleftarrows \mathrm{RH}_{\mathrm{ad}} \\
& \mathrm{RH}_{\mathrm{ad}}+\mathrm{Cu}_{\mathrm{int}}^{++} \rightleftarrows \mathrm{CuR}_{\mathrm{ad}}^{+}+\mathrm{H}_{\mathrm{int}}^{+} \\
& \overline{\mathrm{RH}}_{\mathrm{int}}+\mathrm{CuR}_{\mathrm{ad}}^{+} \longrightarrow \mathrm{CuR}_{2 \mathrm{int}}+\mathrm{H}_{\mathrm{int}}^{+}
\end{aligned}
$$

where the subscript "ad" denotes the interfacially adsorbed species and the subscript "int" denotes the species adjacent to the interface, either on the aqueous side or on the organic side. The bar superscript denotes the organic-phase species. The $\mathrm{CuR}^{+}$ species is formed by the fast reaction between the adsorbed oxime molecules and the copper (II) ions in the aqueous phase. The reaction rate is

$$
\begin{aligned}
N & =k^{\prime}\left[\mathrm{CuR}^{+}\right]_{\mathrm{ad}}[\overline{\mathrm{RH}}]_{\text {in } \mathrm{t}} \\
& =k^{\prime \prime}[\mathrm{RH}]_{\mathrm{ad}}\left[\mathrm{Cu}^{2+}\right]_{\mathrm{int}}[\overline{\mathrm{RH}}]_{\mathrm{int}} /\left[\mathrm{H}^{+}\right]_{\mathrm{int}}
\end{aligned}
$$

A constant value of $[\mathrm{RH}]_{\mathrm{ad}}$, i. e. saturation of the interface, was observed in the range of bulk concentration $\overline{C_{\mathrm{RH}}}>10^{-3} \mathrm{~mol} / \mathrm{dm}^{3}$ for the present oxime in $n$ heptane diluent ${ }^{7}$. The condition of virtually constant interfacial population density of an oxime species is met, except in the special case of very low organic bulk concentration $^{11,12)}$. In this case, the rate may be expressed as

$$
N=k \cdot[\overline{\mathrm{RH}}] \cdot\left[\mathrm{Cu}^{2+}\right] /\left[\mathrm{H}^{+}\right]
$$

This indicates that the extraction rate per unit area is proportional to the copper (II) ion and monomeric oxime concentrations and inversely proportional to the hydrogen ion concentration. The concentrations are 


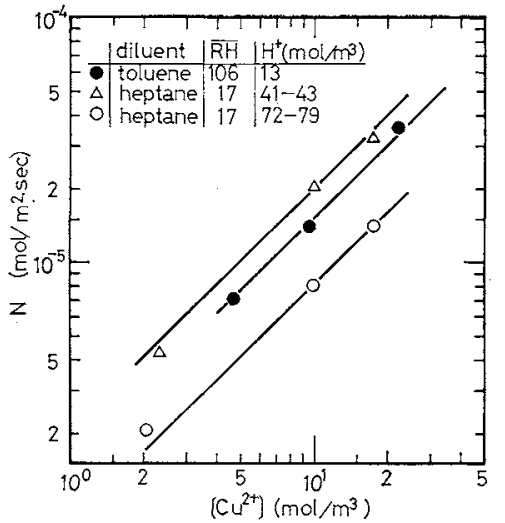

Fig. 3 Effect of copper (II) ion concentration in aqueous bulk phase on extraction rate

not bulk values, however, but those adjacent to the interface, except in the case of negligibly small diffusional resistance in the overall rates. The present reaction scheme expressed by Eq. (2) was previously suggested by Flett ${ }^{3,4}$. They considered, however, that the $\mathrm{CuR}^{+}$species would react with the adsorbed monomer $\mathrm{RH}_{\mathrm{ad}}$ (not with the monomeric oxime in the organic phase).

\section{4 Examination of reaction scheme}

1) Effect of concentrations of species The effect of copper (II) ion concentration on the rate is shown in Fig. 3, which indicates that the reaction is 1 st-order with respect to copper (II) ion concentration. The effect of oxime concentration is shown in Fig. 4, also indicating a 1st-order dependency. The rate data in Figs. 3 and 4 were obtained for a range of hydrogen ion concentrations greater than $8 \times 10^{-3} \mathrm{~mol} / \mathrm{dm}^{3}$ ( $\mathrm{pH}<2.1)$ in the case of $n$-heptane diluent system. These data can be defined as chemical reaction rates, since the values for the observed rates were much smaller than those for the maximum mass transfer rates. The effect of hydrogen ion concentration is shown in Fig. 5, where the dependency is seen to vary with concentration. In the range of high concentration, the rate is inversely proportional to hydrogen ion concentration. The rate data in this range can be defined as chemical reaction rate. With decreasing hydrogen ion concentration, the rate increases more slowly and clearly deviates from a -1 storder dependency. This trend is considered to be caused by the diffusional resistance of hydrogen ion, i. e., by the appreciable difference between the aqueous bulk and interface hydrogen ion concentrations. These data were therefore discounted in the present study and will be discussed in the subsequent publication $^{8}$.

All the data are plotted in Fig. 6, where the data which are assumed to be obtained under the conditions of chemical reaction control are plotted with closed circles. These results are seen to cluster on their

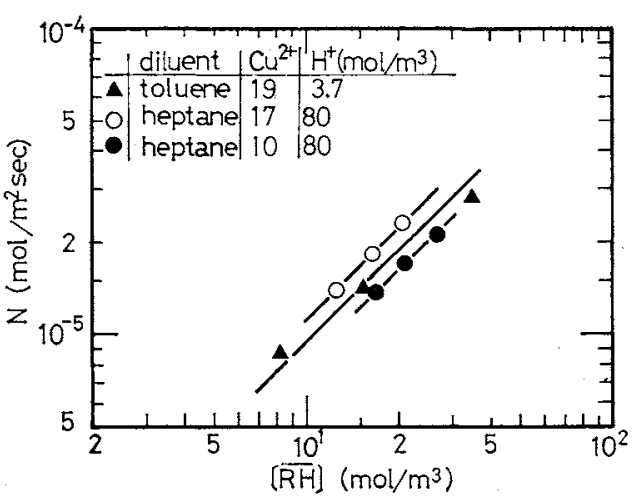

Fig. 4 Effect of monomeric oxime concentration in organic bulk phase on extraction rate

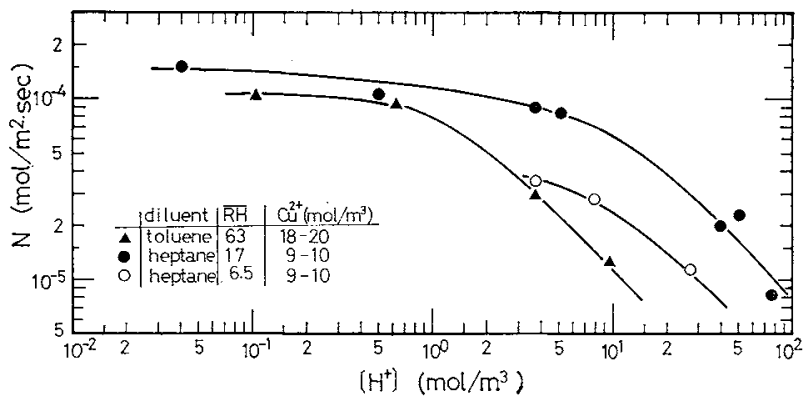

Fig. 5 Effect of hydrogen ion concentration in aqueous bulk phase on extraction rate

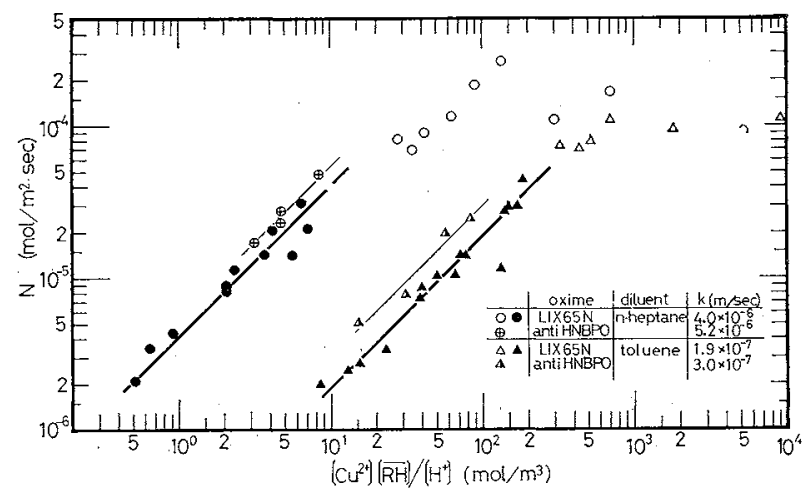

Fig. 6 Correlation of extraction rate of the present work

respective straight line relationships with a slope of 1 for each diluent system. Consequently, the present rates obey the proposed rate expression, Eq. (4). Additional data with anti HNBPO are also plotted. The rates are seen to increase with the purified oxime to differing extents, depending on the diluent. The effect of impurities such as syn HNBPO and p-nonyl phenol, however, is not yet conclusive, since the number of runs with purified oxime is small.

Whewell et al. ${ }^{13)}$ measured the copper extraction rate with LIX64N, using a single-drop technique. Their original plot of the data for a single organic concentration, $20 \mathrm{vol} \%$ LIX64N in Escaid 100, is shown in Fig. 7 with open circles, expressed as a function of total copper concentration. The copper (II) 


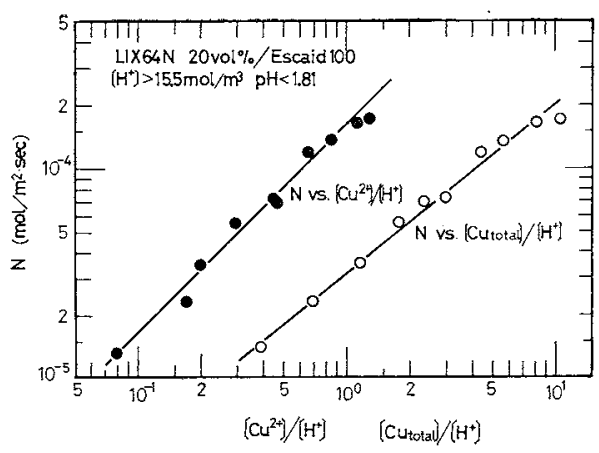

Fig. 7 Correlation of extraction rate of Whewell et al. ${ }^{13)}$

ion concentration for these studies was calculated, as were the present results, for the rate data in the range of high acidity $(\mathrm{pH}<1.8)$, and plotted with closed circles. The resultant rates yield a straight line with a slope of 1 , which indicates that their results can also be correlated by Eq. (4).

2) Effect of diluent on kinetics The rate constant for the $n$-heptane diluent system is nearly 20 times as large as that for the toluene diluent system as shown in Fig. 6. This form of diluent effect on the kinetics has also been observed in other investigations ${ }^{11,13}$. The interfacial concentration of an extractant no longer appears in the form of a relation derived for the rate constant as shown in Eqs. (3) and (4). The interfacial area per molecule of the oxime, anti HNBPO, is estimated as $95 \AA^{2}$ for $n$-heptane ${ }^{7}$ ) and $123 \AA^{2}$ for toluene diluent ${ }^{1}$, and their ratio is as small as 1.3 . It seems therefore quite unlikely for the difference in the rate to be represented by the difference in interfacial area alone. In the equilibrium study ${ }^{7}$ a free monomeric oxime has been suggested, which is the effective oxime with respect to aggregation, aqueous distribution, and copper complex formation. The concentration of this oxime in $n$-heptane has been estimated to be $6-8$ times as large as that in toluene. The differences in the free monomeric oxime content as well as in the interfacial area per oxime molecule may thus contribute to the difference in reaction rate constants.

\section{Conclusion}

The kinetics of the extraction of copper from sulphate media was studied using a single-drop technique. A simple reaction scheme involving the species adsorbed at the interface was proposed, based on the nature of the extractant. This scheme predicts a 1storder dependency for both copper (II) ion and monomeric oxime concentrations and a -1 st-order dependency for hydrogen ion concentration. Assuming the extreme conditions of negligible contribution of the diffusional resistance, the rate data were found to support the reaction scheme proposed.

The extraction rate for the $n$-heptane diluent system was about 20 times as large as that for the toluene diluent system. This diluent effect on the kinetics is likely to result from the interfacial population density of adsorbed monomer and free monomeric oxime content in the organic solution.

\section{Acknowledgment}

The authors gratefully acknowledge the financial support of the Grant-in-Aid for Scientific Research of the Ministry of Education, Science and Culture, Japan (No. 355412, 1978) and experimental assistance of Messrs. T. Kajiwara and N. Ando.

\section{Nomenclature}

$A \quad=$ drop surface area $\left[\mathrm{m}^{2}\right]$

C $\quad$ concentration $\quad\left[\mathrm{mol} / \mathrm{m}^{3}\right]$

$k=$ interfacial reaction rate constant $\quad[\mathrm{m} / \mathrm{sec}]$

$N \quad=$ copper flux $\quad\left[\mathrm{mol} / \mathrm{m}^{2} \cdot \mathrm{sec}\right]$

$V \quad=$ drop volume $\quad\left[\mathrm{m}^{3}\right]$

$\begin{aligned} {\left[\mathrm{Cu}^{2+}\right]=} & \text { copper }(\mathrm{II}) \text { ion concentration in } \\ & \text { aqueous phase } \quad\left[\mathrm{mol} / \mathrm{m}^{3}\right]\end{aligned}$

$[\overline{\mathrm{RH}}] \quad=$ monomeric oxime concentration in organic phase

$\left[\mathrm{mol} / \mathrm{m}^{3}\right]$

$\left[\mathrm{H}^{+}\right] \quad=$ hydrogen ion concentration in aqueous phase $\quad\left[\mathrm{mol} / \mathrm{m}^{3}\right]$

〈Subscripts〉

ad $=$ the species adsorbed at the aqueous-organic interface

bulk $=$ the species in the aqueous or organic bulk phase

int $=$ the species adjacent to the aqueous-organic interface, either on the aqueous side or on the organic side

$\mathrm{RH}=$ oxime extractant

$\mathrm{CuR}_{2} \quad=$ copper complex

$\langle$ Superscript〉

$-\quad=$ the organic species or organic phase concentration

\section{Literature Cited}

1) Al-Diwan, T. A. B. and R. J. Whewell: J. Inorg. Nucl. Chem., 39, 1419 (1977).

2) Fleming, C. A.: N. I. M. Report (Johannesburg), No. 1973 (1976).

3) Flett, D. S.: Account of Chem. Res., 10, 99 (1977).

4) Flett, D. S., D. N. Okuhara and D. R. Spink: J. Inorg. Nucl. Chem., 35, 2471 (1973).

5) Hummelstedt, L.: Paper, 5c, presented at I. S. E. C. '77, Can. Inst. Mining and Metallurgy, Toronto, Sept. 9, 1977.

6) Kojima, T. and T. Miyauchi: Preprint of Gunma Research Meeting, The Soc. Chem. Engrs., Japan, July 13, 1978.

7) Komasawa, I., T. Otake and A. Yamada: J. Chem. Eng. Japan, 13, 130 (1980).

8) idern: ibid., 13, 209 (1980).

9) Komasawa, I. and J. Ingham: Chem. Eng. Sci., 33, 541 (1978).

10) Miller, J. D. and R. L. Atwood: J. Inorg. Nucl. Chem., 35, 2539 (1975).

11) van der Zeeuw A. J. and R. Kok: Paper, 4e, Presented at I.S.E.C. '77, Can. Inst. Mining and Metallurgy, Toronto, Sept. 9, 1977.

12) Whewell, R. J., M. A. Hughes and C. Hanson: ibid., 4a (1977).

13) idem: J. Inorg. Nucl. Chem., 37, 2303 (1975).

14) Whewell, R. J. and M. A. Hughes: ibid., 38, 180 (1976). 